\title{
Measuring the Burden of Disease in Korea
}

This paper provides an overview of the Korean Burden of Disease (KBoD) study, which was the first such study to assess the national burden of disease using disability-adjusted life years (DALYs) in an advanced Asian country. The KBoD study generally followed the approach utilized in the original Global Burden of Disease study (GBD), with the exception of the disease classification and epidemiological data estimation methods used, and the relative weightings of disabilities. The results of the present study reveal that the burden of disease per 100,000 of the Korean population originates primarily from; cancer (1,525 Person Years, PYs), cardiovascular disease (1,492 PYs), digestive disease (1,140 PYs), diabetes mellitus (990 PYs), and certain neuro-psychiatric conditions (883 PYs). These results are largely consistent with those of developed countries, but also represent uniquely Korean characteristics.

Key Words : Mortality; Morbidity; Korea; Burden of Disease

\author{
Seok-Jun Yoon, Sang-Cheol Bae*, \\ Sang-II Lee', Hyejung Chang ${ }^{\ddagger}$, \\ Heui Sug Jo ${ }^{\S}$, Joo-Hun Sung", \\ Jae-Hyun Park", Jin-Yong Lee**, \\ Youngsoo Shin ${ }^{\star \star}$
}

\begin{abstract}
Department of Preventive Medicine, College of Medicine, Korea University, Seoul; Department of Internal Medicine*, Division of Rheumatology, College of Medicine, Hanyang University, Seoul; Department of Preventive Medicine', University of Ulsan, Seoul; Department of Health Sercive Management", Kyung Hee University College of Business Administration, Seoul; Departments of Health Policy and Management ${ }^{8}$; Department of Preventive Medicine" , College of Medicine, Kangwon National University, Choncheon; Research Institute for National Cancer Control and Evaluation National Cancer Center", Goyang; Department of Health Policy And Management* Seoul National University College of Medicine, Seoul, Korea

Received : 30 August 2006

Accepted : 25 October 2006

Address for correspondence

Seok-Jun Yoon, M.D.

Department of Preventive Medicine, College of

Medicine, Korea University, 126-1 Anam-dong 5-ga,

Sungbuk-gu, Seoul 136-705, Korea

Tel : +82.2-920-6412, Fax : +82.2-927-7220

E-mail : yoonsj02@korea.ac.kr

*This research was supported by the Korean Health 21 R\&D Project, Ministry of Health \& Welfare, Republic of Korea (01-PJ1-PG1-OICH10-0007).
\end{abstract}

\section{INTRODUCTION}

In this study, we provide an overview of the Korean Burden of Disease $(\mathrm{KBoD})$ study, which was the first study of its type to assess the national burden of disease in an advanced Asian country, using disability-adjusted life years (DALYs) as measures of disability.

Burden of disease measurements that employ DALYs have been previously utilized by several studies conducted at international and national levels (1-5).

The DALYs concept was initially developed by Murray and Lopez, and was applied for the first time in their authoritative Global Burden of Disease (GBD) project $(6,7)$. This project commenced in the early 1990s and involved researchers in many countries. Moreover, its results have been published in a variety of forms, and researchers in many countries involved, and continue to make, recommendations at the national level and expedite programs that properly address the bur- den of disease issues raised by the $\operatorname{GBD}(3,8)$. In Korea, changing disease trends have been observed over recent years. Specifically, the natures and magnitudes of the threats posed by contagious diseases have receded, whereas the prevalences of non-contagious and chronic diseases have increased (9). Moreover, such changes weigh heavily on the national healthcare system, and make the process of resource allocation a difficult proposition (10).

In this article, we describe some of the key findings of the $\mathrm{KBoD}$ study, which was initiated in the early 2000s and continues today.

\section{MATERIALS AND METHODS}

\section{Overview}

In general, the KBoD study followed the protocols of the 
original GBD study (6). However, the KBoD study differs in terms of the detailed methodology used with respect to; the disease classification and epidemiological data estimation methods used, and the relative weighting of disabilities.

\section{Development of disease classification}

In order to evaluate burden of disease and health care performance, a measure is needed that can be used to quantify scientific health status and to create a systematically organized classification system capable of comprehensibly including every important disease entity.

DALY is a summary measure that represents health status. Using DALYs, WHO arranged ICD codes into 3 major groups, 16 sub-categories, and 93 third-level categories. However, decisions concerning the diseases that should be measured and the units of estimation used are as important as the need for a measurement index. In the present study, we developed a new disease classification that can be used to fully adjust the structure of diseases in Korea and health care performance.

First, we collected and addressed the disease classifications used by WHO and other DALYs yielding procedures. Second, the characteristics of the data sources used to estimate DALYs were scrutinized. Third, the project units of public health activity and public health-related medical services that are in need of the evaluation of public health performances were comprehensibly grasped. Fourth, representative data concerning the disease structure in Korea such as major causes of death and frequent medical service utilizing diseases were investigated. Fifth, based on the above, we developed a new disease classification, which included several added disease entities, and then consulted a team of clinical and public health specialists about the new disease classification system.

Our disease classification is based on the Global Burden of Disease as defined by WHO, and adheres to the principle that a disease classification should be exhaustive and exclusive. On the other hand, considering the ease of approach offered by intervention, and attempt was made to avoid overly detailed disease classifications. In addition, we added diseases included in the Korean national infectious disease surveillance system and the national cancer registration project.

The following diseases (all of which are currently included in the national communicable disease surveillance system) were added to group I of the GBD classification (communicable diseases); herpes genitalia, cholera, typhoid and paratyphoid, shigellosis, mumps, rubella, and chicken pox. In addition, we also added pneumonia and influenza to the classification system. These were added because they were described as 'lower respiratory infections' in the previous GBD classification. Moreover, these diseases are included in the Korean Contagious Disease Prevention Act and thus need to be monitored separately. In group 2 (non-communicable diseases), gallbladder cancer, thyroid cancer, kidney cancer, brain can- cer, and bone and cartilage cancer were added to the 'Malignant neoplasm' subcategory. These disease entities have been under continuous surveillance by the Korean National Cancer Registry project, and are also included in the list of the 236 most frequent causes of death in Korea. Benign brain neoplasm was added to the 'other neoplasms' subcategory, because the disease process and final results associated with brain neoplasms are similar to those of other malignancies. However, neuro-psychiatric diseases were addressed in a somewhat different manner. Instead of simply adding locally important diseases to the current GBD classification, we elected to change the disease classification hierarchy itself.

Consequently, we added a new disease group, which includes 19 disease entities, namely, 8 communicable diseases, 6 neoplastic diseases, and 5 neuro-psychiatric conditions.

\section{Estimation of epidemiologic parameters}

In order to determine the incidences and prevalence of all disease categories, we constructed a large normative cohort, representing the Korean population in 1998 as a cooperative project with the National Health Insurance Corporation of Korea. The Korean National Health Insurance system is an obligatory program that covers all 47 million Koreans. Korean National Health Insurance and related data sources have been previously described in detail (11). We randomly sampled 1,209,693 persons, and stratified them for age into 5-yr age groups, sex, area of residence (Seoul/large city/other), and type of insurance (employee/self-governor/medical aid). The Korean Normative Cohort includes $2.5 \%$ of all Koreans, with and overrepresentation of elderly, to add stability to estimated parameters (Table 1). For the cohort, morbidity and mortality outcomes were followed using data links to the National Health Insurance (KNHI) medical claim and Korean National Statistical Office (NSO) databases during the period 1998 until June 2002. ICD-10 codes which are used by both the $\mathrm{KNHI}$ and NSO were reclassified according to disease classification. All prevalence, incidence, and mortality data were estimated from unit disease classification. Prevalences (per 1,000 persons per year) were calculated after averaging the total number (person-base) of those who had been diagnosed during the follow-up period (1998-2002). Incidences were calculated for 2000 and 2001 after excluding patients who had visited hospital during the first two years (i.e., 1998-1999). To reduce possible over-estimations of morbidity rates caused by using medical claim data, an operational definition for each disease category was made by specialists. For example, cancer cases were counted only when the diagnosis was made in a general hospital, and there were two or more claims for the same disease category. Moreover, all bona vide cancer cases deemed to have required more than one admission event. In terms of mortality, causes of death as reported by the NSO were used as was.

To further refine morbidity rates, 3,678 cases in 9 typical 
Table 1. Characteristics of the representative Korean Normative Cohort

\begin{tabular}{|c|c|c|c|c|c|c|c|c|c|}
\hline \multirow{2}{*}{ Age group } & \multicolumn{3}{|c|}{ Cohort } & \multicolumn{3}{|c|}{ Korean population (2000) } & \multicolumn{3}{|c|}{ Sampling rate } \\
\hline & Male & Female & Total & Male & Female & Total & Male & Female & Total \\
\hline $0-4$ & 42,345 & 38,256 & 80,601 & $1,689,517$ & $1,538,491$ & $3,228,008$ & 2.51 & 2.49 & 2.50 \\
\hline $5-9$ & 47,821 & 41,887 & 89,708 & $1,903,325$ & $1,676,991$ & $3,580,316$ & 2.51 & 2.50 & 2.51 \\
\hline $10-14$ & 41,357 & 37,222 & 78,579 & $1,659,786$ & $1,490,692$ & $3,150,478$ & 2.49 & 2.50 & 2.49 \\
\hline $15-19$ & 48,191 & 45,161 & 93,352 & $1,926,753$ & $1,805,403$ & $3,732,156$ & 2.50 & 2.50 & 2.50 \\
\hline $20-24$ & 50,586 & 48,420 & 99,006 & $2,021,488$ & $1,922,269$ & $3,943,757$ & 2.50 & 2.52 & 2.51 \\
\hline $25-29$ & 56,885 & 53,941 & 110,826 & $2,260,009$ & $2,162,330$ & $4,422,339$ & 2.52 & 2.49 & 2.51 \\
\hline $30-34$ & 57,771 & 55,392 & 113,163 & $2,303,166$ & $2,206,046$ & $4,509,212$ & 2.51 & 2.51 & 2.51 \\
\hline $35-39$ & 56,073 & 53,333 & 109,406 & $2,242,344$ & $2,123,583$ & $4,365,927$ & 2.50 & 2.51 & 2.51 \\
\hline $40-44$ & 53,023 & 50,945 & 103,968 & $2,125,313$ & $2,035,562$ & $4,160,875$ & 2.49 & 2.50 & 2.50 \\
\hline $45-49$ & 38,530 & 37,251 & 75,781 & $1,532,344$ & $1,492,612$ & $3,024,956$ & 2.51 & 2.50 & 2.51 \\
\hline $50-54$ & 30,177 & 29,545 & 59,722 & $1,195,544$ & $1,178,793$ & $2,374,337$ & 2.52 & 2.51 & 2.52 \\
\hline $55-59$ & 25,022 & 26,420 & 51,442 & $1,003,925$ & $1,068,758$ & $2,072,683$ & 2.49 & 2.47 & 2.48 \\
\hline $60-64$ & 20,905 & 24,518 & 45,423 & 840,681 & 971,217 & $1,811,898$ & 2.49 & 2.52 & 2.51 \\
\hline $65-69$ & 14,190 & 19,621 & 33,811 & 568,990 & 777,065 & $1,346,055$ & 2.49 & 2.53 & 2.51 \\
\hline $70-74$ & 8,521 & 14,106 & 22,627 & 336,557 & 568,539 & 905,096 & 2.53 & 2.48 & 2.50 \\
\hline $75-79$ & 5,189 & 9,569 & 14,758 & 209,170 & 387,997 & 597,167 & 2.48 & 2.47 & 2.47 \\
\hline $80-84$ & 3,040 & 6,542 & 9,582 & 98,983 & 218,104 & 317,087 & 3.07 & 3.00 & 3.02 \\
\hline $85-89$ & 1,640 & 5,138 & 6,778 & 34,080 & 104,093 & 138,173 & 4.81 & 4.94 & 4.91 \\
\hline $90-94$ & 879 & 3,322 & 4,201 & 8,957 & 33,216 & 42,173 & 9.81 & 10.00 & 9.96 \\
\hline $95-99$ & 501 & 2,729 & 3,230 & 984 & 6,658 & 7,642 & 50.91 & 40.99 & 42.27 \\
\hline $100+$ & 370 & 3,359 & 3,729 & 172 & 2,048 & 2,220 & $215.12^{\star}$ & $164.01^{*}$ & $167.97^{*}$ \\
\hline Total & 603,016 & 606,677 & $1,209,693$ & $23,962,088$ & $23,770,467$ & $47,732,558$ & 2.52 & 2.55 & 2.53 \\
\hline
\end{tabular}

${ }^{*}$ This age group included those who died between 1998 and 2001, which resulted in oversampling compared with the surviving cross-sectional population.

In an effort to generate a stable epidemiological indicator, weights of $3 \%$ or more were given to age groups of 80 or older as follows: $3 \%$ (80-84), $5 \%$ (85-89), 10\% (90-94), 50\% (95-99), 100\% (100 or older).

disease groups (asthma, epilepsy, stroke, myocardial infarction, herniated intervertebral disk, rheumatoid arthritis, systemic lupus erythematosus, lung cancer, and common surgical conditions) were sampled to validate the KNHI diagnosis based on a medical record review. The concordance rate, which was defined based on "probable cases" and "confirmed cases”, was as low as $52.6 \%$ for asthma (298 acceptable cases out of 567 claims) and as high as $84.1 \%$ for a herniated intervertebral disk (132 acceptable cases out of 157 claims). Several factors were found to be associated with validity, i.e., admission/outpatient, patient age, type of hospital, whether operations were performed or not, total cost, and length of admission. Models were constructed to predict the probability of validity of each case, using the above medical utilization factors. Original prevalence and incidences were recalculated by summing predicted probabilities to yield adjusted morbidity rates. Adjusted morbidity rates were compared to those in previous independent Korean reports when possible.

In order to determine disability duration and average age at onset, we used the DisMod II model (12). Prevalence, incidences, and specific mortality rates were required to estimate disability duration and average age at onset.

\section{Disability weight}

When developing our new classification system, it was found necessary to standardize and quantify a number of social preferences, in order to accurately deduce a set of disability weightings. Multiple measurement methods were used to perform this task, these included person trade off (PTO), time trade off, visual analogue scale, and standard gamble. In the GBD study, however, a protocol was developed based on the PTO measurement scheme (6). In the present study, we selected 16 indicator conditions that fit the Korean context, using three selection criteria as follows: health state should be meaningful in terms of public health, health status is most popular, and health status can be represented on a scale from 0 (representing a good state) to 1 (representing a fatal state of health). After conducting a pilot test of the method using a group of medical students, the PTO protocol was revised in order to render it more appropriate for this study. The 16 indicator conditions are as follows: Chronic back pain, Colon and rectum cancers, Down syndrome, Diabetes mellitus, Peptic ulcer disease, Stomach cancer, Hepatitis B and hepatitis C, Influenza, pneumonia, Unipolar major depression, Dementia, Low birth weight, Schizophrenia, Iron-deficiency anemia, Ischemic heart disease, Epilepsy, and Rheumatoid arthritis.

We then assigned preference weights to selected indicator conditions using the following procedure. First, 30 doctors were grouped into three panels of 10 individuals. The proportions of physicians and preventive medicine specialists were almost same in each panel. 
The first measurement group contained one female doctor and the 2nd and 3rd groups two apiece. Second, each of the three panels was then assigned preference weighting for the 16 indicator conditions, each group taking a different date. Third, panel members took approximately $30 \mathrm{~min}$ to assign preference weightings to each of the health states, using a form developed for this study and by following a coordinator's instructions, were based on PTO1 and PTO2 protocols, respectively. Panel members recorded values to the questions asked in these variants, provided reasons for their answers, and discussed results with other members. After this process had been repeated three times, each of the members provided final values for PTO 1 and PTO2 variants. Forth, disability weightings (DW) were calculated for each of the health states using the PTO values obtained from the panels, as follows: $\mathrm{DW}=1-1000 / \mathrm{PTO} 1=1000 / \mathrm{PTO} 2$. Fifth, median disability weights were recorded on the disability scale, for each of the 16 indicator conditions.

Then, for health states other than those covered by the 16 indicator conditions, each panel member was presented with a set of 37 health states; 30 of which were selected from different categories and 7 from a list of common core diseases. Panel members then assigned preference weights to a total of 123 health states, including the indicator conditions, by interpolation, using the disability scale that had been developed for the indicator conditions.

Finally, the study employed generalizability theory, which is commonly used in the reliability behavior theory, to estimate the relative magnitudes of various components of mea-

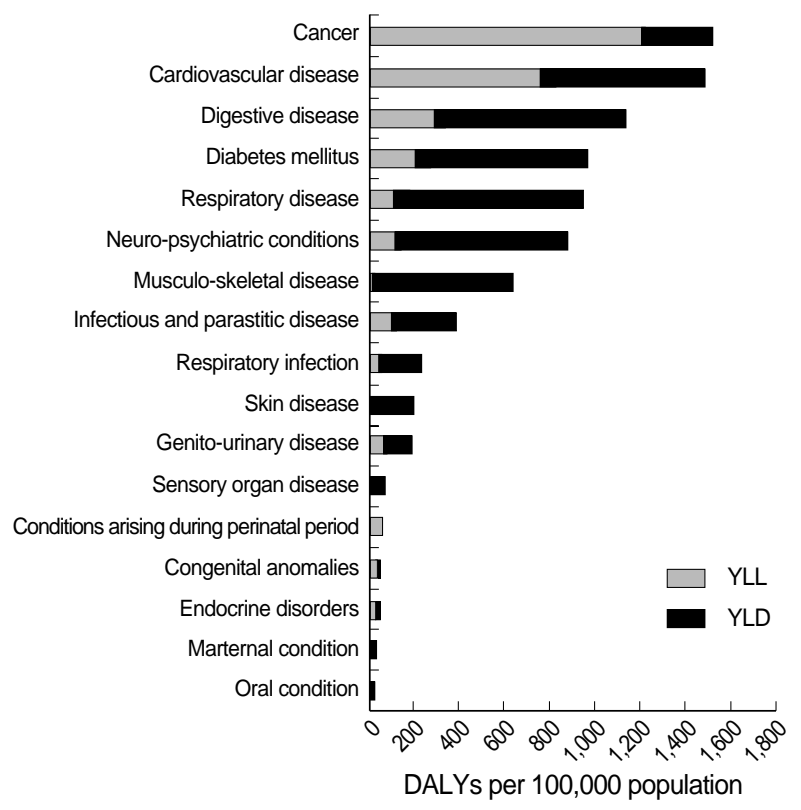

Fig. 1. Burden of disease (years of life lost to premature mortality [YLL], years of life lost due to disability [YLD] and total disabilityadjusted life years [DALYs]) for broad disease groups in Korea in 2002.

Note: DALYs due to injuries are excluded. surements (generalizability study or $\mathrm{G}$ study), by analyzing the reliability coefficient ( $G$ coefficient) and ensuring the desired level of reliability (decision study or D study) $(13,14)$.

The results of this $\mathrm{G}$ study revealed that the degree of contribution made by the 16 indicator conditions was $78.3 \%$. This indicates that the total variance could be explained by the indicator conditions, and that the measurement errors associated with panels and panel members were essentially insignificant. In terms of total variance, 5.2\% was explained by all of the other interactions among the sources of error. The D study showed that the generalizability coefficient was greater than 0.9 in all nine, which suggests a high level of reliability. The generalizability coefficient associated with research design (i.e., indicator conditions, taking triplicorte measurements, 10 respondents for each panel) was also found to be high, at 0.973 .

After completing this process, we estimated DALYs with regard to disease burden in the Korean population.

\section{RESULTS}

The burdens imposed by major disease groups (DALYs lost per 100,000 of the population) are shown in Fig. 1. Cancer was found to be the most prominent cause of disease burden, with a score of 1,525 , followed by cardiovascular diseases

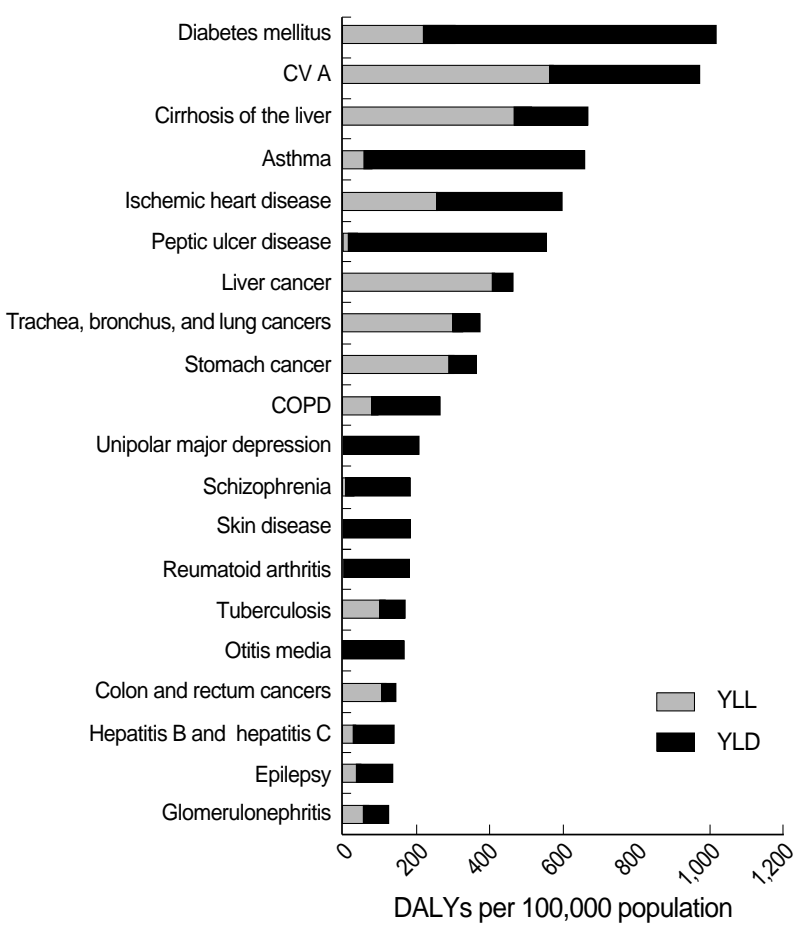

Fig. 2. Top twenty diseases as determined by burden of disease (years of life lost to premature mortality [YLL], years of life lost due to disability [YLD] and total disability-adjusted life years [DALYs]) for major diseases in Korean men in 2002. Note: DALYs due to injuries are excluded. 


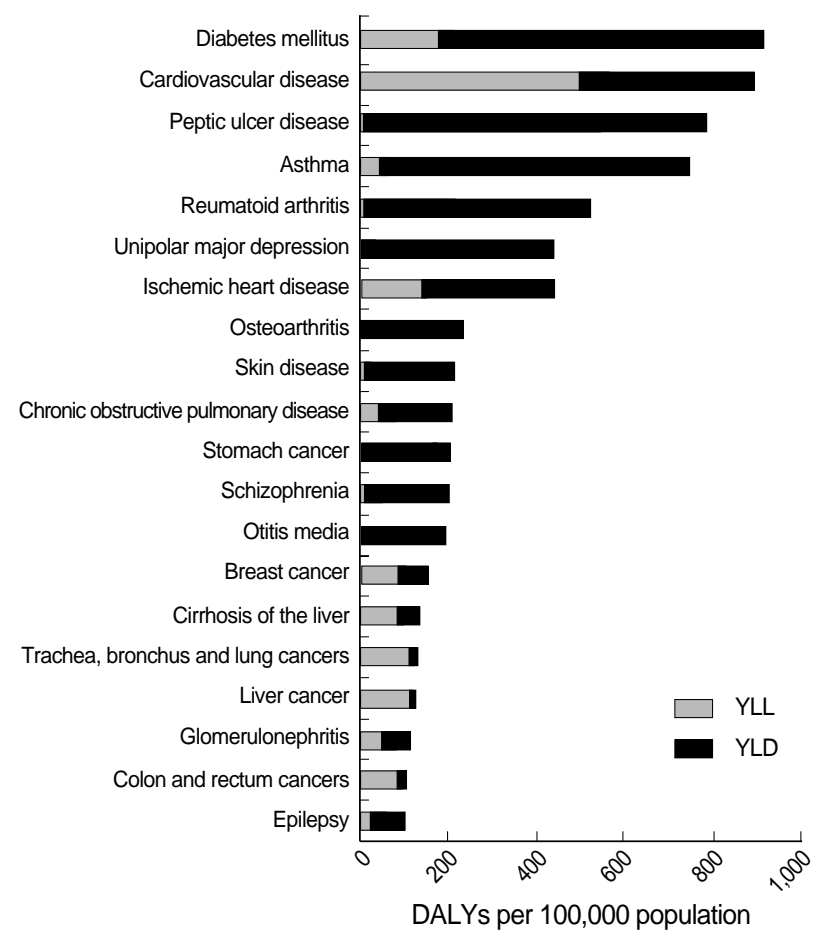

Fig. 3. Top twenty diseases as determined by burden of disease (years of life lost to premature mortality [YLL], years of life lost due to disability [YLD] and total disability-adjusted life years [DALYs]) for primary diseases in Korean women in 2002.

Note: DALYs due to injuries are excluded.

$(1,492)$, digestive diseases $(1,140)$, diabetes mellitus $(970)$, respiratory diseases (951), and neuro-psychiatric conditions (883). The mortality burden of cancer (YLLs lost per 100,000 of the population) was determined to be the leading cause of premature mortality, with a score of 1,222 , and this was followed by cardiovascular diseases (768), digestive diseases (368) and diabetes mellitus (291). Digestive diseases were determined to be the leading causes of disability in Korea (injuries not included), with a YLD of 853 per 100,000 individuals, followed by respiratory diseases (841), neuro-psychiatric conditions (768), and cardiovascular diseases (735) (Fig. 1). A list of the top twenty leading causes of disease burden among men (DALYs lost per 100,000 of the population) is shown in Fig. 2. Diabetes mellitus was found to be associated with the highest burden of disease with 1,020 DALYs, followed by CVA (937), cirrhosis of the liver (671), asthma (663), and ischemic heart disease (601). By individual disease, in men, diabetes mellitus exhibited the highest YLD, at 801 per 100,000 individuals, followed by asthma (610) and peptic ulcer disease (547) (Fig. 2).

The top twenty leading causes of disease burden among women (DALYs lost per 100,000 of the population) are shown in Fig. 3. Diabetes mellitus was associated with the highest DALY rate, with a score of 919 , followed by cardiovascular disease (900), peptic ulcer disease (794), and asthma (755) (Fig. 3). In women, peptic ulcer disease had the highest YLD, at 788 , followed by diabetes mellitus (740) and asthma (710) (Fig. 3).

The burden attributable to unipolar major depression was determined to be twice as high in women than in men, whereas the disability burden associated with cirrhosis of the liver was determined to be 3.7 times as high in men than in women (Fig. 2, 3).

\section{DISCUSSION}

In this study, the authors attempted to measure the burden of disease in Korea, using DALYs. This study represents one of the first examples of such calculations using epidemiologic data and disability weights derived in an Asian country.

DALY, as applied in this study, is a single measurement that consists of a summation of time lost as a result of premature death and time lived under disabling conditions. The primary reason why this indicator has attracted the attentions of researchers in the public health area is that it can be used as a tool for simultaneously measuring the level of death and prevalence $(8,15)$.

The developed classification described above has been found to be a useful tool for many health related studies in Korea and allows comparative studies to be conducted between countries. WHO recommends that a summary measure should correctly reflect the characteristics of a country or region, and that to achieve an intensive study concentrated on a targeted nation should take priority over any other procedures.

The results are expected to provide us with supportive data for evidence-based decision-making and health resource allocation. The results obtained reveal that the burden of disease in Korea originates primarily from cancers, cardiovascular disease, digestive disease, diabetes mellitus, and neuro-psychiatric conditions (Fig. 1). Our findings in this regard are comparable with, but clearly different from, a similar study conducted in Australia (3). In Australia, the burden of disease ranking in men per 100,000 persons using DALY (not including injuries) was found to be cardiovascular disease, cancer, mental disorders, nervous system diseases, and chronic respiratory diseases (3).

Our findings also clearly differ from those of the GBD study for the $\operatorname{EME~}(3,6)$, in which the top ranked diseases using DALYs (not including injuries) were ischemic heart disease, cerebrovascular disease, dementia, tracheal, bronchial and lung cancer, unipolar major depression, and osteoarthritis (3, $6)$, whereas in Korea, the top leading causes were diabetes mellitus, cerebrovascular disease, asthma, peptic ulcer disease, ischemic heart disease, and cirrhosis of the liver.

These results indicate that the burden of digestive diseases, such as, peptic ulcer disease and cirrhosis of the liver, are higher in Korea than in Australia. Korea's higher level of digestive disease burden, especially its higher mortality is explained by a high prevalence of hepatitis B infection and the dietary 
habits of Koreans, who traditionally consume great quantities of salty, highly spiced food $(16,17)$.

The present study has several limitations. First, some degree of uncertainty was introduced by estimating epidemiologic parameters using medical utilization data. Especially, this study did not have exact information regarding utilization data of oriental medicine, pharmacy. However, we found that our results are in line with previous studies on estimated disease incidence in Korea $(8,18)$. Nevertheless, no prevalences or incidences were available for most disease categories, and thus, there remains a possibility that morbidity rates could have been over- or under- estimated (8). Especially, the possibility remains that morbidity rates of older age group could have been overestimated due to the over-sampling method in this study (Table 1). Moreover, the cohort approach used in the present study provided direct estimates and representative morbidity rates for all disease categories, with a certain degree of reliability. Second, computerized data regarding cause of death is not entirely accurate. This is because death certificates are not always issued by physicians, and even when a diagnosis is rendered by a physician, discrepancies sometimes exist between recorded causes of death and actual causes (19). Third, it has also been found to be impossible to determine the burden of disease attributable to injuries in Korea because resulting insurance claims do not contain details of injury type. Moreover, given that the attributable burden of injuries is substantial, this failure to incorporate the influence of injuries into DALY calculations constitutes a major limitation of this study. Finally, the Korean Burden of Disease study is limited by its lack of adjustment for comorbidities, which are likely to affect diseases like diabetes and ischemic heart disease and thus increase the burden of disease (5).

In conclusion, despite the limitations of the data utilized in this study, we are confident that DALY is an appropriate and reasonably accurate tool in the public health area, as has been found in the Netherlands and Australia. Moreover, the present study also provided the basic epidemiologic data to establish an evidence-based health policy.

\section{REFERENCES}

1. Hyder AA, Morrow RH. Applying burden of disease methods in developing countries; a case study from Pakistan. Am J Public Health 2000; 29: 871-7.

2. Melse JM, Essink-Bot ML, Kramers P, Hoeymans N, the Dutch burden of disease group. A national burden of disease calculation: Dutch disability-adjusted life-years. Am J Public Health 2000; 90: 1241-7.

3. Mathers C, Vos T, Stevenson C, Begg SJ. The burden of disease and injury in Australia. Bull World Health Organ 2001; 79: 1076-84.

4. World Health Organization, 2002. Global burden of disease 2002 estimates. Available from: http://www3.who.int/whosis/menu.cfm? path=evidence,burden,burden_estimates,burden_estimates_2002N \&language $=$ english [Sep, 2006]

5. Ljung R, Peterson S, Hallqvist J, Heimerson I, Diderichsen F. Socioeconomic differences in the burden of disease in Sweden. Bull World Health Organ 2005; 83: 92-9.

6. Murray CJ, Lopez AD. The global burden of disease: a comprehensive assessment of mortality and disability from diseases, injuries and risk factors in 1990 and projected to 2020. Boston, Mass; Harvard University Press 1996; 1-98.

7. Murray CJ, Lopez AD. Global mortality, disability, and the contribution of risk factors: global burden of disease study. Lancet 1997; 349: 1436-42.

8. Yoon SJ, Lee HY, Shin YS, Kim YI, Kim CY, Chang HJ. Estimation of the burden of major cancers in Korea. J Korean Med Sci 2002; 17: 604-10.

9. Chang HJ, Myoung JL, Yoon SJ. Preliminary study on national burden of disease. Korea Institute of Health Services Management. 1998.

10. Jeong HS, Kim JK, Lee KS, Shin EC. Priority-setting in expanding the basic benefit package in Korean Health Insurance Scheme. Korean J Health Policy Adm 2004; 14: 34-57.

11. Song YM, Sung J. Body mass index and mortality: a prospective study in a population with low coronary heart disease mortality. Epidemiology 2001; 12: 173-9.

12. Barendregt JJ, Van Oortmarssen GJ, Vos T, Murray CJ. A generic model for the assessment of disease epidemiology: the computational basis of DisMod II. Popul Health Metr 2003; 1: 4-10.

13. Crick JE, Brennan RL. GENOVA: A generalized analysis of variance system (FORTRAN IV computer program and manual). Dochester, Mass.: Computer Facilities, University of Massachusetts at Boston 1982.

14. Stoudhard ME. Disability weights for diseases: a modified protocol and results for a Western European region. Eur J Public Health 2000; 10: 24-30.

15. Barker C, Green A. Opening the debate on DALYs. Health policy plan 1996; 11: 179-83.

16. Korea Institute for Health and Social Affairs. 2001 National Health and Nutrition Survey in Korea. 2001.

17. Lee DH, Kim JH, Nam JJ, Kim HR, Shin HR. Epidemiological findings of hepatitis B infection based on 1998 National Health and Nutrition Survey in Korea. J Korean Med Sci 2002; 17: 457-62.

18. Lee HJ, Yang HK, Ahn YO. Gastric cancer in Korea. Gastric cancer 2002; 5: 177-82.

19. Nam HS, Park KS, Sun BH. A study of the cause-of-death reported on official death rgistry in a rural area. Korean J Prev Med 1996; 29: 227-38. 